\title{
Realization of BP Active Filters using UMLF Structure with Impedance-Tapered Biquads
}

\author{
Dražen Jurišić*, Neven Mijat* and Ivica Mihalić** \\ *University of Zagreb / Signal and Information Processing Department, Unska 3, Zagreb, Croatia \\ ${ }^{* *}$ Siemens d.o.o., Heinzelova 70a, Zagreb, Croatia \\ drazen.jurisic@fer.hr;neven.mijat@fer.hr;ivica.mihalic@siemens.com
}

\begin{abstract}
This paper presents the low-sensitivity realization of band-pass (BP) active resistance-capacitance (RC) filters using "uniform modified leap-frog" (UMLF) structure. Sensitivities with respect to filter passive components are investigated. It is shown how the optimization can be performed in order to achieve minimal sensitivities with relatively small values of $Q-$ factors. Multiple-feedback-structured filters have low sensitivities to passive component tolerances, and additional sensitivity reduction of $2^{\text {nd }}$-order building biquads is achieved by the "impedance tapering" design. As an example, the sensitivity analysis, using Shoeffler sensitivity measure, was performed on a Chebyshev $8^{\text {th }}$-order BP filter. The filters were realized using UMLF and leap-frog (LF) structures, where sensitivity-optimized single-amplifier (SAB) and Sallen and Key (SAK) biquads realize the $2^{\text {nd }}$-order biquads.
\end{abstract}

\section{Introduction}

The realization of BP active- $R C$ filters with transfer functions (TFs) of high order and low sensitivities to passive component variations has been a research interest for many authors in the field [1]-[9]. It is known that the filter structures with multiple feedback loops can posses improved sensitivity properties, compared to the cascade structure of $2^{\text {nd }}$-and $1^{\text {st }}$-order filter blocks [1]-[9].

Particularly low sensitivities have leapfrog (LF) or active ladder structure [Fig. 1(b)], constructed by signal flow graph simulation of passive ladder LC filter. It is known that the sensitivities of the passive ladder LC filters are extremely low [6]. As a consequence, active filters which simulate passive LC networks, have low sensitivities as well. The most important characteristic of the LF structure, in the case of low-pass (LP) filter realization, is that all inner sub-circuits $T_{i}(s)$ are realized using integrators. The first and the last section in the configuration have $1^{\text {st }}$-order transfer functions with real poles. Applying the LP-BP frequency transformation

$$
s \rightarrow\left(s^{2}+\omega_{0}^{2}\right) /(B s),
$$

where $\omega_{0}$ is center frequency and $B$ is the pass-band width, to the LP filter prototype, we construct a BP filter, in which the integrators are transformed into the $\mathrm{BP}$ resonators with infinite pole Q-factor values. The first and last sections have finite pole $Q$ values. From the point of realization complexity this can be a drawback of LF filters, because infinite values of pole $\mathrm{Q}$ factors are not realizable with some types of $2^{\text {nd }}$-order filter sections. For that reason, so-called "uniform modified leapfrog configuration" (UMLF) based on uniform losses of the passive-RLC prototype network has been introduced in [4]. The work in [4] is an extension of the previous work presented in [5] on the "modified leapfrog configuration" (MLF) used for the realization of BP filters with all $2^{\text {nd }}$-order sections having finite values of pole $\mathrm{Q}$ factors.

In this work we first present a simple design procedure of MLF filter based on the ladder LC network simulation with uniform losses. In one example we calculate sensitivities for various values of losses, and demonstrate that by the appropriate choice of those values we can obtain optimal sensitivities and pole $\mathrm{Q}$ values of the filter sections. Additionally, we compare a cascade (CAS) structure as shown in Fig. 1(a), to the filters using LF and UMLF structures as shown in Fig. 1(b) in order to reduce passive components sensitivity. The passive sensitivity is further reduced by the use of low-sensitivity $2^{\text {nd }}$-order biquads of the LF, which are designed using the recently introduced "impedance tapering" design method [10].

\section{The UMLF-structure BP filter realization}

In the design procedure of leap-frog BP filter we start with passive LP prototype of the BP filter, which is realized by a ladder LC network in the Fig. 2(a). Network in Fig. 2(a) and the corresponding procedure belong to the odd-order transfer functions. For the even-order transfer function the procedure is analogous, and will be demonstrated in the design example given below. Let $F(s)$ be its transfer function. For that network, we can write the following equations for nodes and meshes:

$$
\begin{gathered}
I_{0}\left(R_{1}+s L_{1}\right)=U_{\text {in }}-U_{1} \\
U_{1} s C_{1}=I_{0}-I_{2} \\
I_{2} s L_{2}=U_{1}-U_{3} \\
U_{3} s C_{3}=I_{2}-I_{4} \\
\vdots \\
U_{N-2} s C_{(N-1) / 2}=I_{N-3}-I_{N-1} \\
I_{N-1}\left(s L_{(N+1) / 2}+R_{2}\right)=U_{N-2} \\
U_{\text {out }}=U_{N}=I_{N-1} R_{2}
\end{gathered}
$$




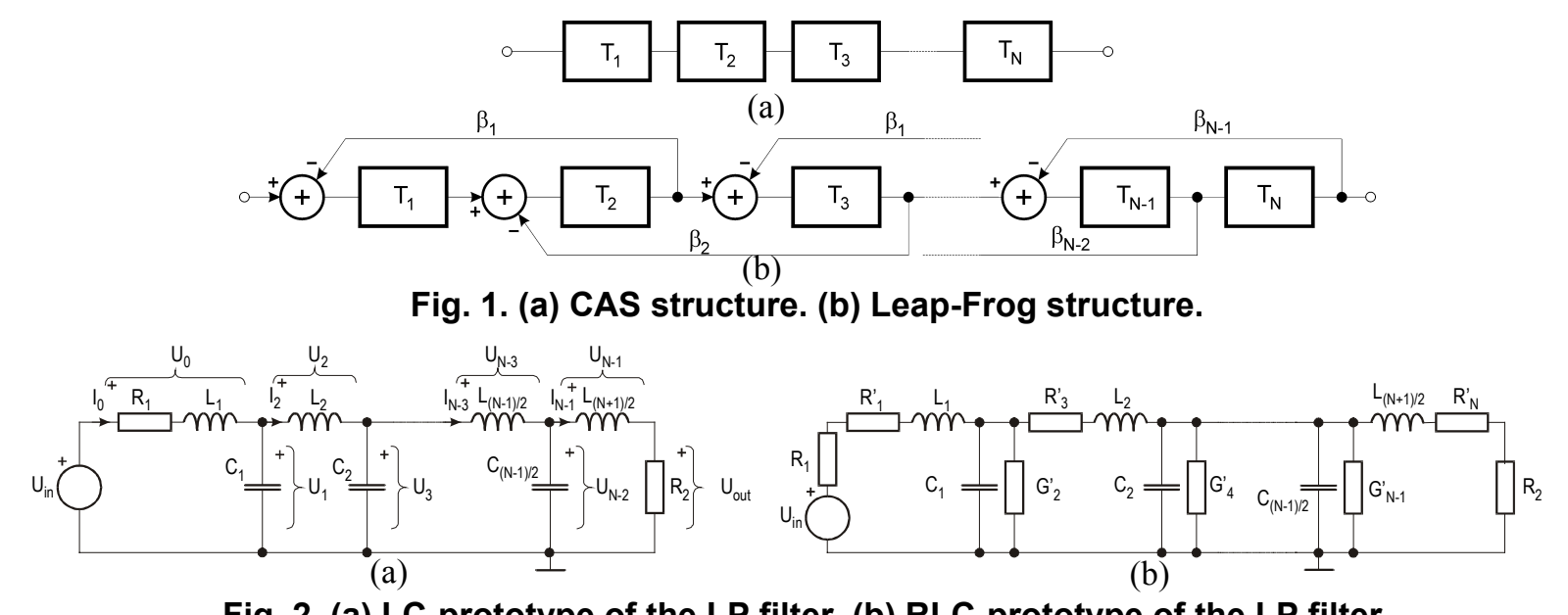

Fig. 2. (a) LC-prototype of the LP filter. (b) RLC-prototype of the LP filter.

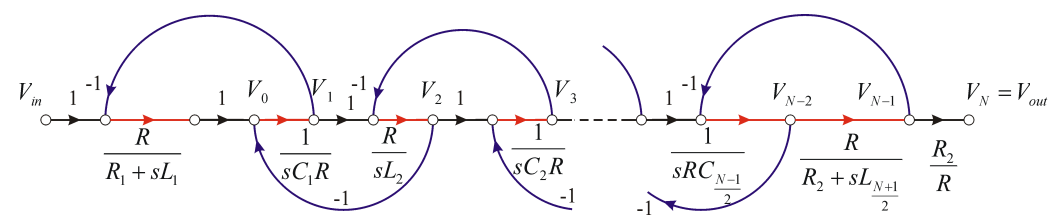

Fig. 3. Signal-flow-graph of LC filter in Fig. 2(a).

If we introduce the following notation:

$$
\begin{gathered}
\quad V_{i}=U_{i} ; \quad i=\text { in }, 1,3, \ldots, N, \\
V_{i}=I_{i} R ; \quad i=0,2,4, \ldots, N-1, \text { out } ;
\end{gathered}
$$

where $R$ represent an arbitrary real constant with dimension $[\Omega]$, then it is possible to present the system of equations (2) by the signal-flow-graph in Fig. 3. We choose the constant $R=1 \Omega$. Using structure in Fig. 1(b) it is possible to realize the signal-flow graph and the transfer function $F(s)$. In that case the $T_{2}, T_{3}, \ldots, T_{N-1}$ are integrators, and $T_{1}$ and $T_{N}$ are the $1^{\text {st }}$-order transfer functions having single real negative poles. In the case of BP filter with $N$ blocks, with LP-BP transformation of variable $s$ we obtain:

$$
\begin{gathered}
T_{i}(s)=\frac{k_{1} \cdot s \omega_{p i}}{s^{2}+\omega_{p i}^{2}} ; \quad i=2, \ldots, N-1 ; \\
T_{i}(s)=\frac{k_{1} \cdot s \cdot \omega_{p i} / q_{i}}{s^{2}+s \cdot\left(\omega_{p i} / q_{i}\right)+\omega_{p i}^{2}} ; i=1, N .
\end{gathered}
$$

From (4a) it can be seen that all inner BP sections of the LF structure have infinite pole $Q$ values. To avoid that, we apply the design procedure proposed in [4], which results in the uniformly modified leap frog (UMLF) structure.

We introduce a new variable $p=s+\delta$ for the LP prototype transfer function $F(s)$, where $\delta$ is a positive and real constant. The function $F(s)$ is transformed into a new function $F_{1}(p)$, which is given by:

$$
F_{1}(p)=F_{1}(s+\delta)=F(s) .
$$

The poles of the transfer function (5) in the complex $p$-plane in comparison to the poles of $F(s)$ (in the complex $s$-plane), are shifted into the imaginary axis direction and parallel to the real axis for the amount $\delta$, as shown in Fig. 4.

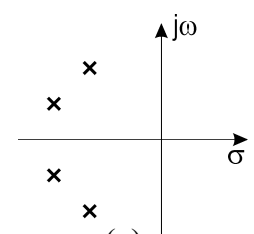

(a)

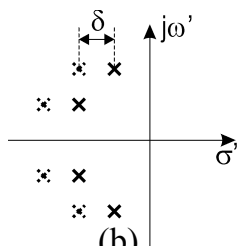

(b)
Fig. 4. TF poles of $4^{\text {th }}-\operatorname{order}(a) F(s)(b) F_{1}(s)$.

The way of calculating the coefficients of the function $F_{1}(p)$ is rather simple, and identical to the way of the parameter calculation in [9]. For the new "predistorted" function $F_{1}(p)$ it is possible to calculate elements of the new LC filter, which has the same form as the filter in Fig. 2(a). The impedances in the series branches of that network are

$$
Z_{L i}=p L_{i},
$$

while in the shunt branches they are

$$
Z_{C i}=1 /\left(p C_{i}\right) .
$$

If we return back to the original variable $s$, they become

$$
\begin{gathered}
Z_{L i}=(s+\delta) L_{i}=s L_{i}+R_{i}^{\prime}, \\
Z_{C i}=1 /\left[(s+\delta) C_{i}\right]=1 /\left(s C_{i}+G_{i}^{\prime}\right),
\end{gathered}
$$

and, as a result, each inductor is transformed into series connection of inductor and resistor, whereas every capacitor into parallel connection of capacitor and resistor. The filter takes on the form shown in Fig. 2(b). Its transfer function differs from the initial transfer function only in a multiplicative constant, a problem easily solved in the active filter realization.

Using the previously described design procedure, starting from passive-RLC filter in Fig. 2(b) we obtain an active leap-frog filter structure of the form shown in Fig. 1(b), with the building blocks transfer function:

$$
\begin{gathered}
T_{1}(s)=\frac{1 / L_{1}}{s+\left(R_{1}+R_{1}^{\prime}\right) / L_{1}} \\
T_{2}(s)=\frac{1 / C_{1}}{s+G_{2}^{\prime} / C_{1}}
\end{gathered}
$$




$$
\begin{gathered}
T_{3}(s)=\frac{1 / L_{2}}{s+R_{3}^{\prime} / L_{2}} \\
\vdots \\
T_{N}(s)=\frac{1 / L_{(N+1) / 2}}{s+\left(R_{N}^{\prime}+R_{2}\right) / L_{(N+1) / 2}}
\end{gathered}
$$

Thus we constructed UMLF filter, which has no integrators, but all $T_{i}(s)$ sections have transfer functions of the $1^{\text {st }}$-order with real and finite poles. Constant $\delta$, although arbitrary, has to be carefully chosen, in order to keep the poles of the transfer function $F_{1}(p)$ in the left half of the $p$-plane. Otherwise the passive LC filter in Fig. 2(b) could not be realized. For the common transfer functions such as Butterworth, Chebyshev, etc., it is possible to circumvent the step in which we calculate the coefficients of $F_{1}(p)$, because we can use the element values of such RLC filters for some discrete values of constant $\delta$ from the tables [11]. Applying the LP-BP transformation to the LP transfer functions $T_{i}(s)$ in (10) we obtain the transfer functions of the filter BP sections. They have the form (4b), i.e. they have finite pole Q factors, which is an advantage compared to the standard LF structure.

\section{3. $2^{\text {nd }}$-order BP biquads realization}

We use Deliyannis or single-amplifier biquad (SAB) and Sallen-and-Key (SAK) biquads (with general impedance scaling factors $r$ and $\rho$ ) as shown in Figs. 5, and 6 , respectively.

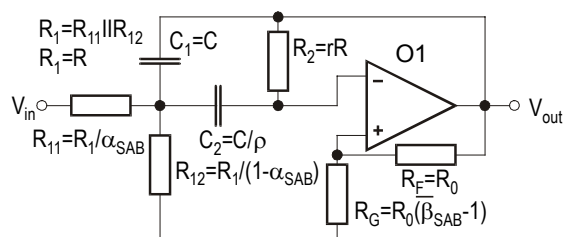

\section{Fig. 5. Deliyannis (SAB) section.}

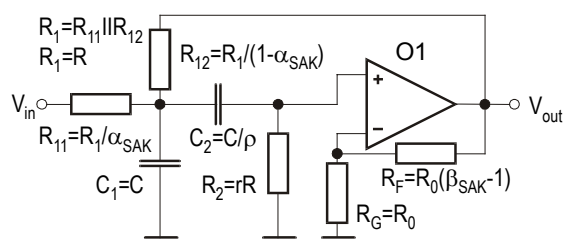

Fig. 6. Sallen \& Key (SAK) section.

The transfer function of the SAB (class-3; negative feedback) band-pass section in Fig. 5 is given by:

$$
T(s)=\frac{V_{\text {out }}}{V_{\text {in }}}=\frac{-\alpha_{S A B} \bar{\beta}_{S A B} \cdot n(s)}{d(s)-\bar{\beta}_{S A B} \alpha_{S A B} n(s)}=\frac{K \cdot\left(\omega_{p} / q_{p}\right) \cdot s}{s^{2}+\left(\omega_{p} / q_{p}\right) \cdot s+\omega_{p}^{2}},
$$

and the transfer function of the SAK (class-4; positive feedback) in Fig. 6 is given by:

$$
T(s)=\frac{V_{o u t}}{V_{\text {in }}}=\frac{\alpha_{S A K} \beta_{S A K} \cdot n(s)}{d(s)-\beta_{S A K}\left(1-\alpha_{S A K}\right) n(s)} .
$$

The filters in Figs. 5 and 6 have the same passive $R C$ network with the numerator and denominator given by:

$$
n(s)=k_{0} \omega_{0} s / q_{0} ; d(s)=s^{2}+\omega_{0} s / q_{0}+\omega_{0}^{2},
$$

where $k_{0}=\frac{R_{2} C_{2}}{R_{1}\left(C_{1}+C_{2}\right)+R_{2} C_{2}} ; q_{0}=\frac{\sqrt{R_{1} C_{1} R_{2} C_{2}}}{R_{1}\left(C_{1}+C_{2}\right)+R_{2} C_{2}}$;

$$
\omega_{0}=\left(R_{1} C_{1} R_{2} C_{2}\right)^{-1 / 2} \text {; and } R_{1}=R_{11} \| R_{12} .
$$

The gain factors $\bar{\beta}_{S A B}=1+R_{G} / R_{F}$ and $\beta_{S A K}=1+R_{F} / R_{G}$ represent the positive-feedback gain factors in class-3 and class- 4 circuits, respectively. Note that the gain factors are related by $1 / \bar{\beta}_{S A B}+1 / \beta_{S A K}=1$. Both circuits are related by the complementary transformation [12], and the optimization conditions for the two, in terms of sensitivity, are identical. The attenuations $\alpha_{S A B}$ and $\alpha_{S A K}$ in both circuits are given by $\alpha=R_{12} /\left(R_{11}+R_{12}\right)$. Comparing (11) and (12) we obtain:

$$
\alpha_{S A B}=\alpha_{S A K} /\left(1-\alpha_{S A K}\right) ; \bar{\beta}_{S A B}=\beta_{S A K}\left(1-\alpha_{S A K}\right) .
$$

Consequently, if all passive elements of the SAK filter are known, we can use the shortcut way to calculate the passive elements of the SAB filter, and vice versa. We just need to calculate the values of the new $\alpha_{S A B}$ and $\bar{\beta}_{S A B}$ from the known $\alpha_{S A K}$ and $\beta_{S A K}$ using (15), or vice versa. In the new filter, passive elements such as $R_{1}, R_{2}, C_{1}$ and $C_{2}$ have the same values, whereas $R_{11}, R_{12}, R_{G}$ and $R_{F}$ readily follow from the new $\alpha$ and $\beta$.

Each high-order (i.e. $n^{\text {th }}$-order; $n>2$ ) symmetrical BP transfer function can be written as a product of $4^{\text {th }}$-order factors if $n=4 k ; k=1,2, \ldots$ and eventually one $2^{\text {nd }}$-order factor if $n=2(2 k+1) ; k=1,2, \ldots$ The $4^{\text {th }}$-order factors have the form:

$$
F_{4}(s)=T_{1}(s) \cdot T_{2}(s)=\prod_{i=1}^{2} \frac{k_{i} \omega_{0 i} s / q_{i}}{s^{2}+\omega_{0 i} s / q_{i}+\omega_{0 i}^{2}},
$$

where $\omega_{01} \omega_{02}=\omega_{0}, q_{1}=q_{2}=q ; \omega_{0}$ is the center frequency and $k_{i}$ is the pass-band gain of a BP filter. Equation (16) represents the cascade realization of a $4^{\text {th }}$-order BP filter. Note, therefore, that e.g. cascaded $8^{\text {th }}$-order BP filter has two pairs of identical pole Q-factors, i.e. $q_{1}=q_{2}$ and $q_{3}=q_{4}$.

In the design process of the SAB and SAK biquads in Figs. 5 and 6, we use the general scaling factors as defined above and given by:

$$
R_{2}=r R_{1} ; C_{2}=C_{1} / \rho .
$$

With known $k_{i}, \omega_{0 i}$ and $q_{i},(i=1, \ldots, n / 2)$ the step-by step design procedure for the $2^{\text {nd }}$-order biquad circuits (SAK and SAB) follow:

(i) Choose capacitor $C$, and impedance scaling factors $\rho=1$, and $r>1$ and then calculate $R$ :

$$
R=\frac{1}{\omega_{0 i} C} \sqrt{\frac{\rho}{r}} .
$$

(ii) Calculate the values

$$
q_{0}=\sqrt{\rho r} /(1+\rho+r), k_{0}=r /(1+\rho+r),
$$

and from (11)-(15) above calculate the $\alpha$ s and $\beta$ s from:

$$
\begin{gathered}
\bar{\beta}_{S A B}=1+(1+\rho) / r-q_{i}^{-1} \sqrt{\rho / r} \\
\beta_{S A K}=\beta_{S A K} \alpha_{S A K}+\bar{\beta}_{S A B}=k_{i} q_{0} /\left(k_{0} q_{i}\right)+\bar{\beta}_{S A B} \\
\alpha=k_{i} q_{0} /\left(k_{0} q_{i} \beta\right) .
\end{gathered}
$$

The above design equations are readily obtained by introducing (17) into (14). Note that when we write $\alpha$ and $\beta$ without subscripts they represent the gain and attenuation of both the SAB and SAK filter sections. Note also that SAB and SAK sections are suitable for 
the realization of both finite and infinite pole Q-factors [see (20)]. For the infinite pole $\mathrm{Q}$ case we have to include the value $k_{i} \omega_{0 i} / q_{i}$ into (21) and (22), instead of $k_{i}$ (see Table 2 below).

(iii) The component values follow from:

$$
R_{1}=R ; R_{11}=\frac{R_{1}}{\alpha} ; R_{12}=\frac{R_{1}}{1-\alpha} ; C_{1}=C ; R_{2}=r R ; C_{2}=\frac{C}{\rho} \text {. }
$$

(iv) Finally, choose $R_{G}$ and compute $R_{F}$ using:

$$
R_{F}=R_{G}\left(\beta_{S A K}-1\right) \text {, }
$$

where, for $\mathrm{SAB}$ filter design, we have:

$$
\beta_{S A K}=\bar{\beta}_{S A B} /\left(\bar{\beta}_{S A B}-1\right) \text {. }
$$

To design the circuit with minimum gain-sensitivityproduct $(\mathrm{GSP})^{1}$, (for both $\mathrm{SAB}$ and $\mathrm{SAK}$ ) we could modify the step $(i)$ into:

( $i$ ') choose $r>1$ and then calculate $\rho$ using:

$$
\rho=r /\left(36 q_{i}^{2}\right) \cdot\left[\sqrt{1+12 q_{i}^{2}(1+1 / r)}+1\right]^{2},
$$

and we could have reduced both passive (by choosing $r>1)$ and active sensitivities. We can calculate the GSP:

$$
\Gamma_{S A K}=q_{i} \beta_{S A K}^{2}\left(1-\alpha_{S A K}\right) \sqrt{r / \rho} ; \Gamma_{S A B}=q_{i} \bar{\beta}_{S A B}^{2} \sqrt{r / \rho} .
$$

Finally, to realize the summing devices in the LF structure in Fig 1(b), we use the circuit having an operational amplifier as in Fig. 7(a). In the block diagram the summing element is shown in Fig. 7(b) and its representation in the SFG is shown in Fig. 7(c).

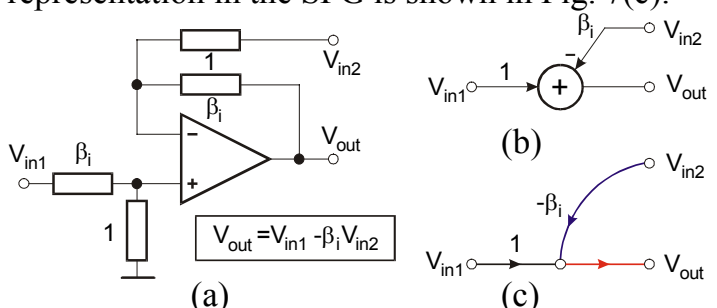

Fig. 7. Opamp realization of a summing device in the LF structure.

\section{Example}

As an example consider an $8^{\text {th }}$-order Chebyshev BP filter with normalized bandwidth $B=1$, center frequency $\omega_{0}=1$, and the pass-band ripple $R_{p}=0.1 \mathrm{~dB}$. Its magnitude $\alpha(\omega)=20 \log |F(j \omega)|[\mathrm{dB}]$ is shown in Fig. 9(a). We proceed according to the UMLF filter design procedure given above. A passive-RLC LP filter prototype of the UMLF BP filter is shown in Fig. 8. It was derived from the ladder-LC filter with resistor $R_{L}$ at the output. For the above filter example, normalized element values of a passive-RLC LP prototype (with the cut-off frequency $\omega_{g}=1$ ) for 4 different values of the constant $\delta$ from the tables in [11] are given in the Table 1.

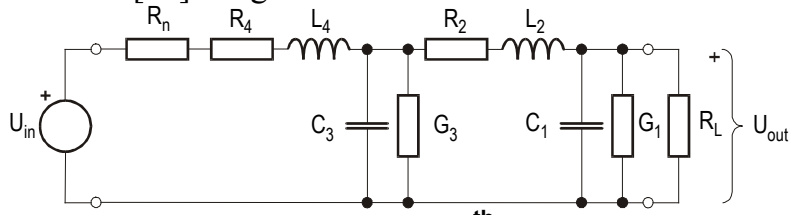

Fig. 8. RLC prototype of $4^{\text {th }}$-order Chebyshev LP filter.

1 The GSP gives a measure of a filters magnitude sensitivity to the open-loop gain $(A)$ variation of the active component [14].
Table I Passive RLC UMLF filter elements (LP prototype).

\begin{tabular}{|l|l|l|l|l|}
\hline \hline$\delta$ & 0.0 (LF) & 0.0333 & 0.05 & 0.1 \\
\hline \hline$R_{L}$ & 1.0 & 1.0 & 1.0 & 1.0 \\
\hline$G_{1}$ & 0.0 & 0.019953 & 0.031175 & 0.07124 \\
\hline$C_{1}$ & 2.3545 & 0.5986 & 0.6235 & 0.7124 \\
\hline$R_{2}$ & 0.0 & 0.041497 & 0.063445 & 0.13481 \\
\hline$L_{2}$ & 0.7973 & 1.2449 & 1.2689 & 1.3481 \\
\hline$G_{3}$ & 0.0 & 0.049403 & 0.074785 & 0.15466 \\
\hline$C_{3}$ & 2.6600 & 1.4821 & 1.4957 & 1.5466 \\
\hline$R_{4}$ & 0.0 & 0.03951 & 0.057595 & 0.1035 \\
\hline$L_{4}$ & 0.3623 & 1.18530 & 1.1519 & 1.035 \\
\hline$R_{n}$ & 0.5 & 0.0 & 0.0 & 0.0 \\
\hline
\end{tabular}

The transfer functions of LP prototypes and the resulting BP filter building blocks in UMLF structures [obtained by the application of the LP-BP frequency transformation as in (1)] are given by:

$$
\begin{gathered}
T_{1}(s)=\frac{1 / L_{4}}{s+\left(R_{n}+R_{4}\right) / L_{4}} \rightarrow \frac{B / L_{4} \cdot s}{s^{2}+B\left(R_{n}+R_{4}\right) / L_{4} \cdot s+\omega_{0}^{2}} \\
T_{2}(s)=\frac{1 / C_{3}}{s+G_{3} / C_{3}} \rightarrow \frac{B / C_{3} \cdot s}{s^{2}+B G_{3} / C_{3} \cdot s+\omega_{0}^{2}} \\
T_{3}(s)=\frac{1 / L_{2}}{s+R_{2} / L_{2}} \rightarrow \frac{B / L_{2} \cdot s}{s^{2}+B R_{2} / L_{2} \cdot s+\omega_{0}^{2}} \\
T_{4}(s)=\frac{1 / C_{1}}{s+\left(G_{L}+G_{1}\right) / C_{1}} \rightarrow \frac{B / C_{1} \cdot s}{s^{2}+B\left(G_{L}+G_{1}\right) / C_{1} \cdot s+\omega_{0}^{2}}
\end{gathered}
$$

Note that equations (28) are also valid for $\mathrm{LF}$ structure $(\delta=0)$ and they have $R_{i}=0(i$ odd $)$ and $G_{j}=0(j$ even). If we introduce component values from Table 1 into (28) we obtain parameters of the UMLF and LF $2^{\text {nd }}$-order BP filter building biquads $T_{i}(s)$ presented in Table 2. The parameters of the cascaded filter sections are presented in Table 2, as well. Note that the gain values $k_{i}$ and feedback values $\beta_{i}(i=1, \ldots, 4)$ are optimized by the method given in [13], to provide maximum dynamic range.

\begin{tabular}{|c|c|c|c|c|c||}
\hline \hline \multicolumn{7}{||c|}{ Table II Parameters of CAS, MLF and LF filter structures } \\
realizing the 8th-order Chebyshev BP TF
\end{tabular}




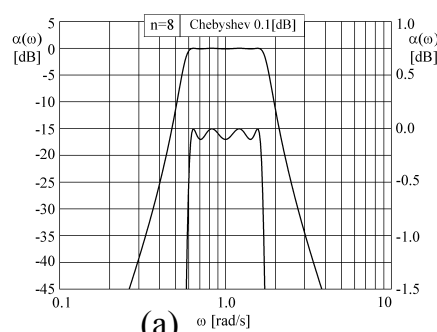

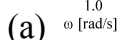

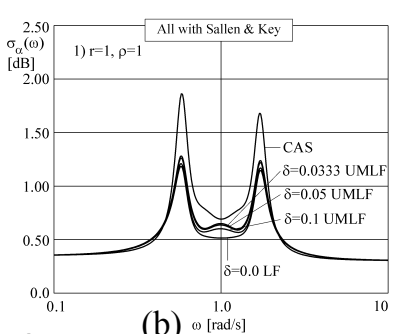

(b) $\frac{1.00}{1 \text { [rads] }}$

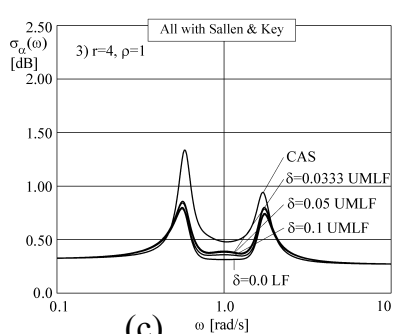

(c) $\underset{\text { of }[\text { [rads] }}{\log }$

Fig. 9. (a) Frequency response of the $8^{\text {th }}$-order Chebyshev BP filter example. (b) Shöffler sensitivity of filters examples: CAS to LF with design strategy 1. (c) CAS to LF with design strategy 3.

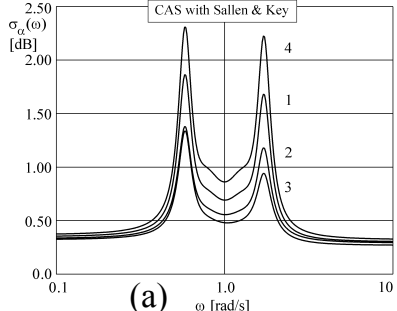

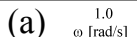

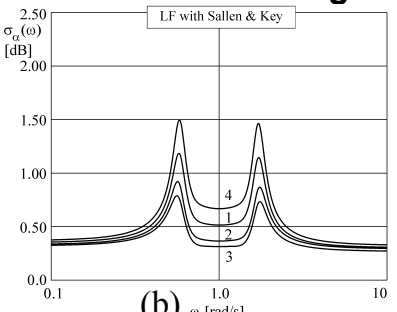

(b) $\stackrel{1.0}{1.0}$

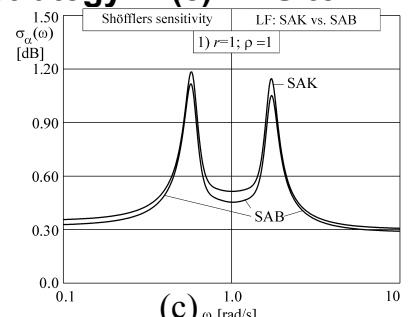

(c) $\stackrel{\substack{1.0 \\ \text { [rad } s]}}{(\text { a }}$

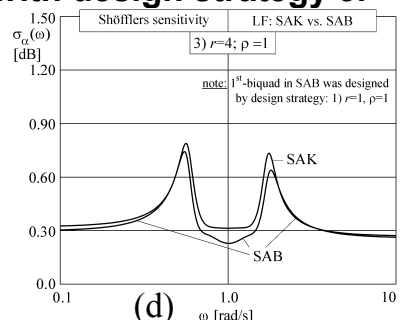

(d) $\underset{\substack{1.0 \\ \text { w [rads] }}}{10}$

Fig. 10. Shöffler sensitivity of filters examples. (a) CAS. (b) LF. (c)-(d) Min. sensitivity SAK vs. SAB.

In what follows we design the $8^{\text {th }}$-order BP filter using all structures in Table 2 having building biquads realized with SAK as in Fig. 6. In the design we apply various ways of impedance tapering to the SAK biquads, in the CAS, UMLF and LF structures, i.e.: 1) $r=1, \rho=1$; 2) $r=4, \rho=4$; 3) $r=4, \rho=1$; and 4) $r=1, \rho=4$.

In a sensitivity analysis we assume the relative changes of the biquad resistors and capacitors to be uncorrelated normal random variables, with zero-mean and $1 \%$ standard deviation. The feedback-resistor sensitivity influence was not considered.

First, we demonstrate that the sensitivities of the UMLF filter depend on the chosen value of the constant $\delta$ (see [4]). In that sense, the parameter $\delta$ can be used for optimization of the sensitivities of the filter, in that we try to find its value, for which the minimum sensitivities of the filter with relatively low pole Q values of the sections will result. Figs. 9(b) and (c) show how the sensitivities of the UMLF filter change for different values of the constant $\delta$. The results of the sensitivity analysis of all 5 filters examples, in Table 2, are presented. The corresponding standard deviation $\sigma_{\alpha}(\omega)[\mathrm{dB}]$ (related to the Shöfflers sensitivities) of the variation of the $\log$ gain $\Delta \alpha=8.68588 \Delta|F(j \omega)| /|F(j \omega)|$, with respect to passive elements, for the design strategies no. 1) and no. 3) are shown in Figs. 9(b) and (c), respectively.

In researching the influence of the constant $\delta$ value we start from the UMLF minimum value of $\delta=0.0333$ for which the curve $\sigma_{\alpha}(\omega)$ takes on smaller values than those for CAS realization in the whole pass band. By further increase of the $\delta$ value the sensitivities decrease further, thus $\sigma_{\alpha}(\omega)$ for the curve with $\delta=0.05$ has lower values than for the curve with $\delta=0.033$. We conclude that with $\delta$ increase the sensitivities decrease. From Table 2 it can be seen that with increase of the constant $\delta$, pole $\mathrm{Q}$ factors of the UMLF sections decrease, as well. For $\delta=0.1$ they are lower than the minimal pole Q factors values of the cascaded filter. This is an important advantage of the UMLF type of filter over cascaded filter and over LF filter. Together with pole Q decrease the corresponding sensitivities of UMLF filters decrease, as well. This is logical since sensitivities to component tolerances of "medium-Q" filters are proportional to the filters pole Q [14]. In the special case when $\delta=0$ we have the LF structure, which has the minimum sensitivity. In the stop band the sensitivities of all analyzed structures are higher and near each to other [4].

The standard deviation $\sigma_{\alpha}(\omega)[\mathrm{dB}]$ for CAS and LF structures for all design cases from 1) to 4) are shown in Figs. 10(a) and (b), respectively. Observing the standard deviation in the Fig. 10(a) one can investigate which is the proper impedance tapering design strategy of biquads in the cascade (CAS), in order to reduce the overall sensitivity of the $8^{\text {th }}$-order BP filter. It can be seen that the "ideally impedance-tapered" biquads (no. 2) and even more the "partially-tapered" biquads with equal capacitors (no. 3), decrease sensitivity with respect to component variations, compared to the nontapered standard circuit (no. 1).

Further desensitization is obtained by an application of a negative feedback, which has a LF structure as in Fig. 1(b). The sensitivity curves of LF filters, designed with all four impedance-tapering strategies applied to $2^{\text {nd }}$-order biquads, are shown in Fig. 10(b). The sensitivity curves in Fig. 10(b) have the same order as those in the CAS case in Fig. 10(a), i.e. 4, 1, 2, 3, starting from the curve with highest and ending with the lowest sensitivity. Note, that all LF curves in Fig. 10(b) are lower than those for the CAS structure in Fig. 10(a).

Finally, the Schöffler sensitivities of the LFs with SAB and SAK section for the design strategies 1) and 3) are presented separately in Fig. 10(c) and (d), respectively. In both cases $\mathrm{SAB}$ biquads produce slightly lower sensitivity than SAK, although both circuits have the same passive-RC networks. Note that the $1^{\text {st }}$ biquad in LF realization had to be realized by

\footnotetext{
2 The terms "ideal tapering" and "partial tapering" are used according to the definition in [10].
} 
non-tapered SAB (i.e. using design strategy no. 1), because of its very low pole Q-factor value.

\section{Conclusions}

In this work a procedure for the realization of $\mathrm{BP}$ active filters using UMLF structure was presented, by which all $2^{\text {nd }}$-order building sections have finite values of pole-Q factors. The structure is based on the calculation of passive RLC filters that have uniform losses. It was demonstrated that the sensitivities of this structure are somewhat higher than those of the wellknown LF structure, but they are still much lower than those of the standard cascade structure. Changing the amount of the loss in the passive RLC prototype $\delta$, it is possible to optimize parameters of the sections in order to obtain minimum sensitivities together with obtaining the low values of pole Q-factors [4]. Finally, the leapfrog structure (LF) which has $\delta=0$ (lossless prototype) has minimum sensitivity.

By application of a recently introduced "impedance tapering" design method of $2^{\text {nd }}$-order biquads, their passive sensitivities are additionally decreased. In this paper, we therefore present a twofold sensitivity reduction, one by introduction of negative feedback (application of the LF structure) and the other by the proper design of the $2^{\text {nd }}$-order sub-circuit building blocks in LF. The optimum design strategy that reduces passive sensitivities is tapering the resistors and keeping the capacitor values equal.

By application of the complementary transformation it is demonstrated that the realization of optimized biquads using SAK also provides optimized SAB biquads, whereas the latter realization shows slightly lower passive sensitivities of the $8^{\text {th }}$-order LF filter.

\section{References}

[1] K. R. Laker, R. Schaumann, M. S. Ghaussi, "Multiple loop feedback topologies for the design of low sensitivity active filters", IEEE Trans. on Circ. Syst., CAS-26, pp. 1-21, Jan. 1979.

[2] F. E. J. Girling, E. F. Good, "Active filters, part 12: The leapfrog or active ladder synthesis," Wireless World, pp. 341345, July 1970.

[3] F. E. J. Girling, E. F. Good, "Active filters, part 13: Application of active ladder synthesis," Wireless World, pp. 445-450, Sept. 1970.

[4] N. Mijat, "Realization of BP Active Filters using UMLF Structure," in Proc. of $29^{\text {th }}$ Yugoslav Conference ETAN 1985, Niš, Serbia \& Montenegro, Vol. 3, June 3-7, 1985, pp. 57-64. [5] K. R. Laker, M. S. Ghaussi, J. J. Kelly, "Minimum sensitivity active (leap frog) and passive ladder bandpass filters," IEEE Trans. on Circ. Syst., CAS-22, pp. 670-677, Aug. 1975.

[6] H. J. Orchard, "Inductorless filters," Electron. Letters, vol. 2, pp. 224-225, June 1966.

[7] N. Mijat, G. S. Moschytz, "Multiple-critical-pole coupled active filters," Int. Journal Circuit Theory and Appl., vol. 12, pp. 249-268, July 1984.

[8] N. Mijat, Low-sensitivity structures in realization of active filters, Doctoral Thesis, Univ. of Zagreb, Croatia, Oct. 1984 (in Croatian).

[9] J. Tow, "Design and evaluation of shifted-companion form active filters," Bell Syst. Tech. Journal, vol. 54, no. 3, pp 545-568, March 1975.

[10] G. S. Moschytz, "Low-sensitivity, low-power, active- $R C$ allpole filters using impedance tapering," IEEE Trans. on CAS-II: vol. CAS-46, no.8, pp. 1009-1026, Aug. 1999.

[11] L. Weinberg, Network analysis and synthesis, McGrawHill, New York, 1962.

[12] G. S. Moschytz and P. Horn, "Optimizing two commonly used active-filter building blocks using the complementary transformation," Electronic Circuits and Systems, vol. 1, no. 4, pp. 125-132, July 1977.

[13] D. J. Perry, "Scaling Transformation of MultipleFeedback Filters", Proc. IEE, vol.128, pp.176-179, Aug. 1981.

[14] G. S. Moschytz and P. Horn, Active Filter Design Handbook, Chichester, U.K.: Wiley 1981. 